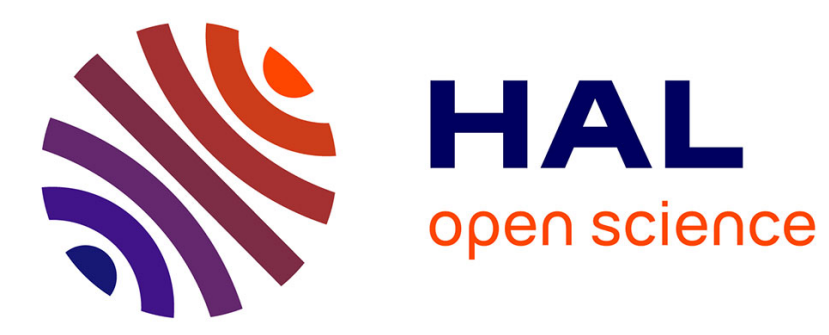

\title{
L'ÉDUCATION THÉRAPEUTIQUE : ENTRE SAVOIRS COMPLEXES, FORMATEURS, APPRENANTS HÉTÉROGÈNES ET CONTEXTES PLURIELS
}

Maryvette Balcou-Debussche

\section{To cite this version:}

Maryvette Balcou-Debussche. L'ÉDUCATION THÉRAPEUTIQUE: ENTRE SAVOIRS COMPLEXES, FORMATEURS, APPRENANTS HÉTÉROGÈNES ET CONTEXTES PLURIELS. Recherche en soins infirmiers, 2012, http://www.cairn.info/revue-recherche-en-soins-infirmiers-20123-page-45.htm. 10.3917/rsi.110.0045 . hal-01153725v1

HAL Id: hal-01153725

https://hal.science/hal-01153725v1

Submitted on 11 May 2016 (v1), last revised 2 Mar 2021 (v2)

HAL is a multi-disciplinary open access archive for the deposit and dissemination of scientific research documents, whether they are published or not. The documents may come from teaching and research institutions in France or abroad, or from public or private research centers.
L'archive ouverte pluridisciplinaire HAL, est destinée au dépôt et à la diffusion de documents scientifiques de niveau recherche, publiés ou non, émanant des établissements d'enseignement et de recherche français ou étrangers, des laboratoires publics ou privés. 


\section{L'ÉDUCATION THÉRAPEUTIQUE : ENTRE SAVOIRS COMPLEXES, FORMATEURS, APPRENANTS HÉTÉROGÈNES ET CONTEXTES PLURIELS}

Maryvette Balcou-Debussche

Association de recherche en soins infirmiers (ARSI) | « Recherche en soins infirmiers »

2012/3 N 110 I pages 45 à 59

ISSN 0297-2964

Article disponible en ligne à l'adresse :

http://www.cairn.info/revue-recherche-en-soins-infirmiers-2012-3-page-45.htm

Pour citer cet article :

Maryvette Balcou-Debussche, «L'éducation thérapeutique : entre savoirs complexes, formateurs, apprenants hétérogènes et contextes pluriels », Recherche en soins infirmiers 2012/3 ( $\left.N^{\circ} 110\right)$, p. 45-59.

DOI 10.3917/rsi.110.0045

Distribution électronique Cairn.info pour Association de recherche en soins infirmiers (ARSI).

(C) Association de recherche en soins infirmiers (ARSI). Tous droits réservés pour tous pays.

La reproduction ou représentation de cet article, notamment par photocopie, n'est autorisée que dans les limites des conditions générales d'utilisation du site ou, le cas échéant, des conditions générales de la licence souscrite par votre établissement. Toute autre reproduction ou représentation, en tout ou partie, sous quelque forme et de quelque manière que ce soit, est interdite sauf accord préalable et écrit de l'éditeur, en dehors des cas prévus par la législation en vigueur en France. Il est précisé que son stockage dans une base de données est également interdit. 


\title{
L'éducation thérapeutique : entre savoirs
} complexes, formateurs, apprenants hétérogènes et contextes pluriels

\author{
Therapeutic education : between complex knowledge, educators, heterogeneous \\ learners and plural contexts
}

\section{Maryvette BALCOU-DEBUSSCHE}

Maître de conférences, Laboratoire LCF-Icare, Université-IUFM de La Réunion Membre de la Commission Scientifique du réseau national UNIRéS

\section{Cette conférence a été présentée lors des journées d'Etude de l'ARSI 2012}

\begin{abstract}
RÉ S U MÉ
L'éducation thérapeutique interroge les liens entre les sciences médicales et les sciences humaines et sociales à travers des dimensions d'ordre épistémologique, praxéologique et éthique. Le développement de l'éducation thérapeutique questionne la complexité de différents contextes en France et à l'étranger, dont la nécessité d'intégrer les particularités et les variations. La présente contribution interroge les fondements théoriques et les conditions de développement de situations d'apprentissage intégratives impliquant des malades chroniques et des formateurs ayant bénéficié de socialisations différentes. Développés en France et à l'étranger, les dispositifs analysés ont été élaborés à partir d'une analyse préalable des spécificités des savoirs en jeu, des représentations des apprenants et d'agencements pouvant produire des résultats auprès d'individus dont l'hétérogénéité et la diversité sociale et culturelle ont été pensées d'emblée. Plus de dix mille personnes ont déjà bénéficié de cycles éducatifs. Les résultats mettent en évidence le développement de nouvelles organisations et dynamiques sociales. Les analyses invitent à une réflexion sur les dimensions sociale et contextuelle de l'apprentissage, l'accès aux savoirs des personnes malades chroniques et les possibilités de développement par divers formateurs, dans différents lieux.
\end{abstract}

Mots clés : éducation thérapeutique, apprentissage, contexte, formation, hétérogénéité.

A B S T RACT

Therapeutic patient education questions the links between medical and social sciences through epistemological, praxeological, and ethical issues. Its development in France and abroad necessary invites to consider the complexity of the particularities and variations of numerous contexts. The present contribution examines the theoretical foundations and the conditions required for the development of integrative learning situations, which involve both persons with chronic diseases and educators who have beneficiated from diverse socialisations. These learning situations have been worked out across three distinct stages: prerequisite analysis of specific knowledge at stake and of learners' representations preceding the thorough design of procedures that can yield results with heterogeneous individuals of various cultural and social origins. More than ten thousands persons have beneficiated from these learning situations courses. Results underline the development of emerging social dynamics and organisations beyond the learning process. These analysis invite to the reflection on social and contextual dimensions of learning, on the access to knowledge for persons with chronic diseases and the opportunities to develop the approach by diverse educators and trainers from various areas.

Key words : self-management, learning, social context, education, heterogeneity.

Pour citer l'article :

BALCOU-DEBUSSCHE M. L'éducation thérapeutique : entre savoirs complexes, formateurs, apprenants hétérogènes et contextes pluriels. Recherche en soins infirmiers, septembre $2012 ; 110: 45-59$ 


\section{INTRODUCTION}

Les difficultés auxquelles le praticien éducateur en santé se heurte permettent de considérer d'emblée que l'éducation à la santé et l'éducation thérapeutique questionnent plusieurs dimensions. La première est d'ordre épistémologique : est-il légitime et concevable de rapprocher les sciences médicales et les sciences humaines et sociales en sachant qu'il s'agit là de deux grands champs scientifiques qui mobilisent chacun des concepts, des méthodes, des types d'analyses et des façons de produire du sens fort différentes ? La seconde est d'ordre praxéologique : comment réussir à intégrer la complexité de ce qui est en jeu, tant du côté des savoirs que du côté des apprenants et des contextes dans lesquels ces derniers sont appelés à agir ? Enfin, d'un point de vue éthique et déontologique, comment réussir à conseiller sans imposer, à prévenir sans faire peur, à accepter que l'apprenant fasse un choix différent de celui que l'éducateur avait escompté ou aurait aimé voir se réaliser?

Bien qu'elles soient en développement à l'heure actuelle en France, les recherches qui s'intéressent à l'éducation à la santé et à l'éducation thérapeutique sont encore trop peu nombreuses (Deccache, 1995) [1] ; (Manderscheid, 2006) [2]. Même si les expériences menées sur différents terrains rendent compte de modèles, de méthodologies et de pratiques diversifiées à l'appui (Foucaud \& al. 2010) [3], les pratiques éducatives se réfèrent essentiellement aux modèles behavioriste ou constructiviste (Billon, 2000) [4]. Centrée sur la prédiction des comportements et leur contrôle par différents types de renforcements, l'approche behavioriste repose sur un lien de causalité entre l'information et le changement d'attitude ainsi que sur l'hypothèse d'une relative rationalité des conduites des individus (Skinner, 1953) [5]. À l'inverse, le constructivisme conduit à intégrer les mécanismes de la construction de la connaissance (Piaget, 1979) [6], le rôle des interactions et du langage dans l'apprentissage (Vygotski, 1978) [7], ainsi que la nécessité de varier les activités de l'apprenant et les formes de manifestation des savoirs en vue d'une meilleure construction des concepts (Roger, 2003) [8]. Les pratiques mises en œuvre se différencient ainsi par l'importance et la place qu'elles accordent ou non à un accompagnement psychologique et social prenant en compte les besoins, les désirs et les motivations des individus. Les actions éducatives oscillent ainsi entre un modèle biopsychosocial de la santé qui tient compte des facteurs sociaux, contextuels et personnels influant sur la santé, et un modèle biomédical de la santé qui travaille les connaissances à construire sur les organes, la santé et la maladie sans nécessairement prendre en compte les spécificités des individus et de leurs environnements.

Face à ces questionnements et distinctions manifestes, la présente contribution interroge la pertinence et la faisabilité d'une élaboration conceptuelle intégrative en éducation thérapeutique. La perspective retenue conduit à mobiliser des travaux scientifiques validés dans les deux champs que sont les sciences médicales et les sciences humaines et sociales, en intégrant de façon constructive les questionnements cités précédemment. La mise à l'épreuve de cette construction théorique sur des terrains et contextes différenciés est analysée en tenant compte des résultats obtenus dans différentes structures d'éducation et de prévention en France et à l'étranger. Les atouts et les limites de l'approche sont questionnés, de même que les perspectives de développement, y compris du côté des professionnels non soignants.

\section{CADRE DE RÉFÉRENCE}

\section{La dimension sociale de la maladie}

Plusieurs travaux scientifiques mettent en évidence le fait que les problèmes liés au développement rapide des pathologies chroniques ne relèvent pas uniquement du champ médical. La maladie reste le plus souvent appréhendée sur un versant anatomo-physiologique par les professionnels de santé alors que pour la personne malade, il s'agit avant tout d'un événement psychologique et social (Herzlich, 1986) [9] ; (Laplantine, 1993) [10] ; (Lawton, 2003) [II]. Les sociétés qui comme l'île de La Réunion - premier terrain de nos travaux scientifiques ont connu des changements profonds durant les cinquante dernières années (Wolff \& Watin, 20I0) [12], constituent dès lors des espaces qui permettent de questionner les décalages et les tensions qui se retrouvent au cœur des interrogations sur l'éducation thérapeutique. Dans la société réunionnaise, les nombreux changements n'ont pas épargné les façons de manger, de bouger et de se soigner, bouleversant ainsi les rapports de la population à la nature, au corps, à la religion et au monde médical (Balcou-Debussche, 20I0) [13]. Dans un contexte pluriel où se mêlent des populations de diverses origines culturelles, la personne malade ne peut pas être considérée comme étant uniquement porteuse d'une pathologie : c'est avant tout un être social, en dynamique et en interaction perpétuelle avec d'autres individus de la famille, du monde du travail ou du voisinage, dans des environnements géographique, culturel et économique spécifiques. Comme dans beaucoup d'autres lieux, les malades chroniques réunionnais sont pris entre plusieurs tensions et ils apportent des réponses thérapeutiques qui vont des attitudes de repli à des consommations de soins aussi excessives que défaillantes, à l'attente de l'action sociale ou politique pour améliorer les conditions d'amélioration de l'existence, au refus des changements demandés par les médecins ou au nomadisme sous forme d'un « marronnage médical et thérapeutique » qui rend autant compte de fortes insécurités que de la volonté réelle de trouver des solutions aux problèmes posés par la maladie (Balcou-Debussche, 2006) [14]. S'il est important de considérer que l'ensemble de ces tensions constitue la toile de fond sur laquelle se tissent les relations entre les soignants et les personnes malades, il faut aussi souligner le fait que ces tensions ne sont pas spécifiques au terrain réunionnais. Elles apparaissent ailleurs, avec des variations qui rendent compte de l'expression de rapports 


\section{L'éducation thérapeutique : entre savoirs complexes, formateurs, apprenants hétérogènes et contextes pluriels}

inégalitaires et de relations fort différentes à la maladie, à l'autre et au monde social en général.

Menée entre 2003 et 2005 à La Réunion, une recherche ethnosociologique annexée à une étude épidémiologique (Debussche, Rollot, Le Pommelet \& al. 2012) [15] a permis d'analyser et de mettre en évidence la complexité des pratiques de 42 personnes diabétiques vues dans deux contextes différents : I'hôpital et le domicile. Les analyses ont permis de saisir la diversité des parcours de malades chroniques qui, après avoir bénéficié de séances éducatives complémentaires dans le cadre hospitalier, se retrouvaient confrontés à la gestion « ordinaire » de leur maladie chronique quelques jours ou semaines après le retour chez eux. L'étude a été travaillée en croisant des données complémentaires, dont de nombreuses observations in situ de séances éducatives à l'hôpital, des entretiens avec les $\mathbf{4 2}$ personnes à leur retour à domicile (trois à quatre semaines après l'hospitalisation), des observations de pratiques menées six à huit semaines plus tard au domicile des personnes (Balcou-Debussche, 2006) [14]. Parmi les principaux résultats mis en évidence par cette recherche ethnosociologique, l'un des aspects saillants porte sur le fait que les personnes malades chroniques se sentent plutôt bien lors de leur séjour à l'hôpital, notamment parce qu'elles sont « prises en charge » par un corps médical et paramédical dévoué qui prend soudain le relais dans la gestion de la maladie. Soulagées du poids que représente la maladie, les personnes malades chroniques se retrouvent ainsi « en suspension des réalités » ordinaires, ce qui leur permet d'ailleurs de se restaurer sur plusieurs plans (alimentaire, psychologique) sans que soit nécessairement travaillée leur identité de malade chronique (Balcou-Debussche \& Debussche, 2009) [16]. Lors des séances d'éducation à l'hôpital, les personnes ont peu accès à la parole et la compréhension des enjeux relatifs à la santé et à la maladie est incertaine. Au final, de retour à leur domicile, les malades reprennent souvent les habitudes qui sont les leurs, en lien avec des rapports sociaux le plus souvent inchangés et avec des contextes sociaux et économiques parfois difficiles. Le travail réalisé en éducation n'apporte donc pas les bénéfices escomptés, même si les apprenants en sont globalement satisfaits et si le personnel soignant y consacre beaucoup d'énergie et de volonté.

\section{Contextualiser les situations éducatives}

Si l'on tient compte des contextes dans lesquelles elles s'inscrivent, les actions éducatives sont donc à penser dans leur dimension éminemment sociale (Durkheim, 1922) [17]. Certes, l'action d'éducation ou de formation met a minima en

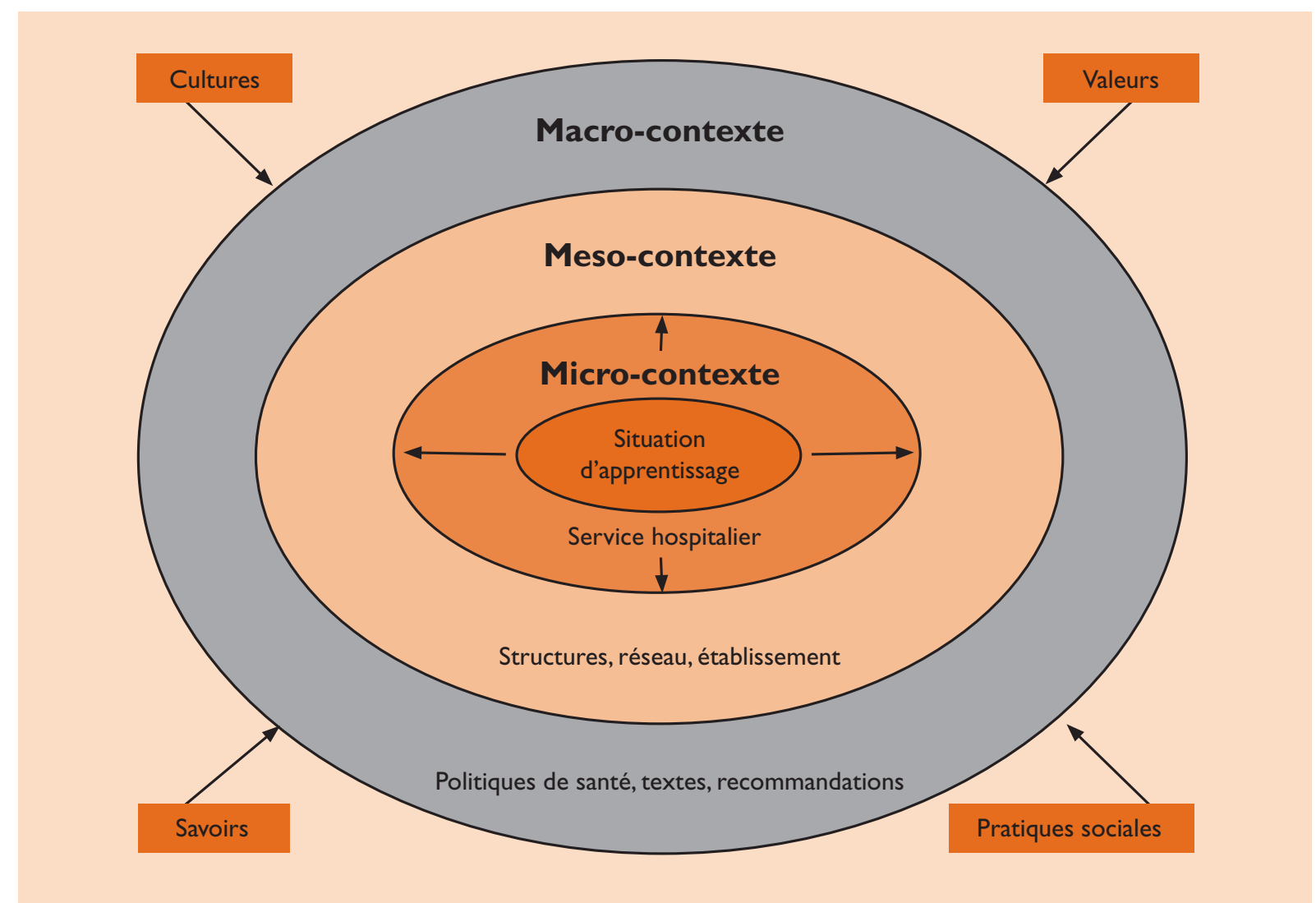

Figure 1 : Modèle contextuel des situations d'apprentissage 
jeu un triangle composé d'un formateur, d'un apprenant et de savoirs (Houssaye, 2000) [18], mais il ne faut pas oublier pour autant qu'un tel triangle pédagogique s'inscrit lui-même dans plusieurs cercles concentriques contextuels qui exercent une influence importante sur les modalités de la rencontre entre les savoirs, les apprenants et les formateurs. Ces cercles se situent a minima à trois niveaux : au centre, le niveau micro avec tout ce qui relève du micro-situationnel (la séance éducative, le groupe d'éducation), au-delà le niveau méso avec la prise en compte de la dimension périphérique (le service, l'hôpital) et enfin le niveau macro qui prend en compte le contexte des politiques de santé, les recommandations et la société dans ses dimensions religieuses, culturelles, linguistiques, économiques. Au centre, là où se déclinent les situations d'enseignement-apprentissage, la rencontre « savoirs-formateur-apprenant » subit les forces extérieures en même temps qu'elle en est la traduction, si bien que les frontières des recherches restent à déterminer au cas par cas, obligeant ainsi à « une contextualisation scientifique sans cesse renouvelée » (Tupin, 2006) [19].

La rencontre éducative s'effectue presque toujours dans un contexte qui n'est pas celui de l'individu (l'hôpital, le cabinet du médecin, la structure associative). La question qui reste posée dès lors est de savoir comment penser et construire cette rencontre alors que le contexte est largement constitutif de la capacité ou non de l'individu à développer les conduites adaptées à une amélioration de sa santé ou à la gestion de sa maladie. Plutôt que de considérer seulement les pratiques des individus sous l'angle de leurs caractéristiques psychologiques ou de leur histoire particulière, ces pratiques sont analysées ici comme relevant d'une combinaison entre des dispositions individuelles qui sont à mettre en lien avec les modes de socialisation auxquels les individus ont été confrontés, et les configurations sociales dans lesquelles ils sont amenés à évoluer (Lahire, 2002) [20]. Cet éclairage permet de comprendre, tout au moins pour partie, que certaines situations éducatives ne produisent pas les résultats attendus, (par exemple lorsque la situation ne permet pas d'activer les dispositions de l'individu), mais il permet aussi de regarder la situation éducative comme une configuration sociale particulière qui n'a pas beaucoup à voir avec les configurations ordinaires dans lesquelles la santé de l'individu est mise en jeu. Dès lors, la difficulté est importante puisque l'action éducative ne peut presque jamais s'effectuer dans les contextes ordinaires de l'individu, alors qu'il apparaît pourtant nécessaire de négocier les propriétés de l'environnement dans lequel toute action s'inscrit (Bril, 2002) [2I]. L'action éducative devrait permettre à l'apprenant de travailler sur l'identification des affordances des environnements en l'amenant à percevoir ce que l'environnement permet dans une situation donnée, notamment en l'aidant à étudier l'ensemble des configurations possibles et en identifiant les éléments à contrôler (Gibson, 1977) [22]. Les recherches sur les pratiques d'enseignementapprentissage et leurs contextes devraient dès lors permettre une plus large prise en compte des variables contextuelles, notamment en vue d'une progression dans la connaissance et l'efficacité des processus d'enseignement et d'apprentissage (Tupin, 2006) [19].

\section{De la transmission des savoirs à leur construction}

Dans la relation triangulaire entre les savoirs, les apprenants et les formateurs, l'un des trois éléments est mis en coulisses à l'hôpital puisque l'on se situe surtout dans la relation enseignant-savoir avec une dominante d'enseignement sur un mode transmissif, voire prescriptif (Balcou-Debussche, 2003) [23] ; (Balcou-Debussche, 2004) [24]. Or, certaines conditions doivent être réunies pour que la transmission soit efficace : le formateur et les apprenants doivent avoir à peu près la même façon de produire du sens, de se poser les questions, de pratiquer le langage et de produire du raisonnement (Giordan, 1998) [25]. En outre, la fonction de transmission comporte toujours un déni implicite de la place du sujet dans sa propre éducation, ce qui conduit le formateur à un échec professionnel et social quand il est englué dans une relation de transmission adossée à une logique strictement disciplinaire (Meirieu, 1997) [26]. II n'empêche que le paradigme de la transmission reste fortement prégnant et que bon nombre de situations d'éducation thérapeutique sont encore modelées par une façon de concevoir la rencontre éducative qui laisse peu de place aux profonds décalages qui existent entre les socialisations dont ont bénéficié les soignants et celles de bon nombre de malades chroniques.

Le changement de paradigme qu'impose le passage de la transmission à la construction des savoirs oblige dès lors à prendre en compte plusieurs dimensions : les représentations individuelles (Giordan, 1998) [25], la conception et l'agencement de dispositifs d'apprentissage (Roger, 2003) [8], la dimension contextuelle de l'apprentissage et la perception que le malade peut avoir des atouts ou des contraintes de son environnement. La perspective n'est plus de penser la personne malade chronique comme quelqu'un qui doit seulement devenir " compliant », mais comme une personne qui a bénéficié de socialisations différentes de celle du soignant, qui a des projets et des valeurs différentes tout en faisant l'expérience singulière de la gestion de sa maladie chronique au quotidien. Plutôt que d'être toujours considéré en termes de manques de motivation, de volonté ou de compétences, l'apprenant est alors pris en compte dans la complexité de ses rapports aux savoirs et à la maladie. En référence au socioconstructivisme, une place particulière est accordée à l'activité des apprenants dans la situation d'apprentissage, dont l'activité qui s'exerce à travers les pratiques langagières (Bautier, 1995) [27]. Y compris lorsque les personnes ne maîtrisent pas suffisamment la lecture et l'écriture, le formateur est amené à penser l'apprenant comme étant capable d'exercer un travail sur les savoirs qui sont en jeu, mais aussi sur les rapports à sa maladie, à sa santé, aux autres et au monde.

Sur le plan praxéologique, la complexité et la pluralité de ces exigences fait apparaître une difficulté importante lorsque 


\section{L'éducation thérapeutique : entre savoirs complexes, formateurs, apprenants hétérogènes et contextes pluriels}

l'éducation thérapeutique est développée par les soignants. En effet, ces derniers se retrouvent confrontés à deux logiques différentes : la logique de transmission des savoirs à travers laquelle ils ont été formés et la logique d'appropriation par la construction qu'ils devraient pouvoir développer afin de permettre aux apprenants de s'approprier des connaissances complexes (Gravois Lee et Garvin, 2003) [28]. Cette difficulté est notamment à mettre en lien avec le fait qu'en France, les soignants des structures hospitalières restent largement centrés sur la dimension curative et technique des soins aux personnes (Rothier-Bautzer, 2002) [29]. En médecine libérale, les professionnels de santé entretiennent une « illusion d'éducation » qui ne favorise pas nécessairement le développement de pratiques éducatives diversifiées : peu formés à l'éducation, ils réduisent le plus souvent cette dernière à la seule dimension informative et explicative (Balcou-Debussche \& Debussche, 2008) [30].

L'ensemble de ces éléments montre les difficultés auxquelles les malades chroniques, mais aussi les soignants se retrouvent confrontés lorsque, dans un même lieu (la séance éducative) ils sont supposés réussir ensemble une rencontre productive, à partir de laquelle des résultats sont escomptés. La question n'est pas simple et les travaux scientifiques dont nous disposons en France ne permettent pas d'éclairer suffisamment la complexité de ce qui se passe dans les situations éducatives elles-mêmes, mais aussi et surtout en dehors, y compris dans des contextes pluriels où il ne s'agit plus de penser l'individu à travers la seule transmission de recommandations faites pour un patient standard ou idéal (Hughes, 2003) [3I]. L'enjeu consiste, à l'inverse, à penser la complexité à travers « l'homme pluriel » pensé lui-même en termes non linéaires et non permanents (Lahire, 1998) [32], mais aussi à envisager la différence et I'hétérogénéité non pas comme un problème, mais comme une source de richesses qu'il s'agit d'apprendre à explorer, à analyser et à valoriser. Cet apprentissage est donc à faire du côté du soignant qui doit nécessairement faire évoluer sa posture de professionnel. Mais il doit aussi se réaliser du côté de la personne malade chronique qui doit saisir les enjeux de sa participation active à un processus en construction, lequel oblige à des réinterprétations progressives bien plus qu'il n'invite à se soumettre à des prescriptions ou des recommandations.

Enfin, tout comme l'éducation à la santé, l'éducation thérapeutique ne peut pas être envisagée à travers la seule construction de connaissances : c'est aussi et surtout pouvoir construire des compétences psychosociales qui vont permettre d'agir de façon opportune en situation (Berger, 2004) [33]. La seule maîtrise de savoirs procéduraux appris comme des règles ne suffit pas pour être efficace car la compétence met en jeu plusieurs dimensions : les savoirs (connaissances intellectuelles, représentations), l'agir (capacités à mettre en œuvre) et des formes de reconnaissance sociale validées et partagées par différents acteurs (Foucaud \& Balcou-Debussche, 2008) [34]. La compétence renvoie également à des situations complexes qui conduisent l'individu à gérer des variables hétérogènes pour résoudre des problèmes qui échappent à des situations difficiles à référer à une seule discipline (Meirieu, 197) [26]. Dans le cas de la maladie chronique, l'individu doit gérer l'incertitude du pourquoi, notamment d'un point de vue médical, ainsi que ce qui touche directement à sa vie : les symptômes, les traitements aux effets incertains, les durées hypothétiques (Bury, 1982) [35]. L'enjeu consiste alors à développer chez l'individu, qu'il soit en bonne santé ou malade, des capacités d'action qui permettront d'être efficace dans des situations complexes qui relèvent tout autant de dimensions sociales, culturelles, économiques et cognitives que du domaine médical (Lawton, 2003) [I I].

\section{Action, décision et rapports de pouvoir}

L'éducation thérapeutique impose enfin que soit intégrée la décision d'actions par l'apprenant lui-même, sans que cette décision s'exerce sous l'autorité du professionnel de santé ou du professionnel éducateur (Falkum et Forde, 200I) [36]. Afin de donner un réel pouvoir de décision à l'apprenant, le formateur doit nécessairement prendre de la distance par rapport à l'approche téléologique par finalités et intentions majoritairement développée dans les pratiques d'éducation thérapeutique. Cette exigence suppose que l'apprenant dispose de la connaissance de tous les éléments en présence pour pouvoir décider, puis agir. L'apprentissage doit à la fois s'exercer grâce à l'accompagnement du professionnel éducateur et indépendamment de lui puisque l'individu est amené à développer ses conduites de façon singulière, au quotidien, dans le contexte qui est le sien. Si le travail sur les pôles politique et cognitif de l'autonomie à laquelle les apprenants doivent accéder est incontournable (Lahire, 200I) [37], une réflexion importante reste à mener pour que le savoir détenu par le professionnel éducateur puisse être approprié par l'apprenant sans que persiste la relation de dépendance qui structure la plupart des actions d'éducation. Dans les situations d'apprentissage, les apprenants n'échappent pas aux conflits sociocognitifs liés à la confrontation de leurs représentations avec la rationalité scientifique (Lautier, 200I) [38], mais les recherches montrent aussi que les individus sont plutôt enclins à vouloir réduire les dissonances de leurs cognitions (Festinger, 1957) [39] et à conserver une image positive d'eux-mêmes (Tajfel, 1972) [40]. Dès lors, il n'est pas nécessaire d'envisager la situation éducative en termes de rapports de force et de conflits, d'autant que les situations éducatives impliquent des personnes malades qui, de ce fait, ont déjà un nombre important de tensions à gérer dans leur vie ordinaire, sur le long terme.

\section{PROBLÉMATISATION ET MÉTHODOLOGIE}

\section{A la recherche de réponses intégratives}

L'analyse non exhaustive des principaux éléments constitutifs de ce cadre théorique permet de situer l'intérêt et les enjeux d'une imbrication étroite entre la connaissance et la 
compétence, l'apprentissage et sa dimension contextuelle ainsi que les exigences de la décision d'actions, dans le cadre particulier de l'éducation thérapeutique. C'est ainsi que sur le plan praxéologique, les situations d'apprentissage se sont construites progressivement comme une forme de réponse intégrative aux problèmes posés par la complexité de la question eu égard aux différences de socialisations des individus et aux particularités des contextes dans lesquels les personnes sont amenées à négocier leur parcours de malade chronique. Les situations d'apprentissage (appelées « nids d'apprentissage ») ont été construites à partir d'une dialectique permanente entre la dimension théorique et la dimension pratique et de nombreuses expérimentations ont été menées avec une large diversité d'individus, et dans des contextes différents. Les situations prennent appui sur les apports du socioconstructivisme tout en considérant les contextes dans lesquels les individus évoluent au quotidien, de même que l'exigence de la décision d'actions à mener par l'individu concerné. Plutôt que de réduire la complexité des éléments en jeu dans la santé ou la maladie ou de n'en travailler que quelques aspects les uns après les autres, les situations d'apprentissage s'efforcent d'incorporer l'ensemble des éléments en faisant le choix de les travailler de façon clarifiée et constructive.

Le « nid d'apprentissage » peut alors être défini comme une situation centrée sur la construction de savoirs dans laquelle les savoirs travaillés in situ vont permettre à l'apprenant de prendre des décisions relatives à la gestion de sa santé, de modifier éventuellement ses pratiques ordinaires tout en tenant compte de ce que l'apprenant est en tant qu'individu singulier, en interrelation avec les autres ou avec son environnement. Le travail s'exerce ainsi sur deux grands champs. D'une part, le champ des connaissances avec la présence d'un espace d'analyses libres et d'un espace d'analyses encouragées qui va être fonction de la qualité de conception de la situation et de la capacité du formateur à éduquer la capacité d'attention de l'apprenant en vue de nouvelles élaborations. D'autre part le champ de l'action avec un espace de liberté qui ne doit pas faire oublier que la décision d'action appartient toujours à l'individu. En parallèle, un espace d'actions encouragées conduit le formateur à travailler sur les dispositions individuelles de l'apprenant, en l'aidant à prendre conscience des atouts et des contraintes de son environnement. Le nid d'apprentissage ne peut pas être considéré comme une véritable proposition écologique puisqu'il est construit par le professionnel éducateur pour favoriser les constructions de savoirs et de compétences psychosociales, mais il s'en inspire largement et cherche à s'en rapprocher le plus possible.

Chaque situation d'apprentissage propose une rencontre constructive entre les conceptions des apprenants et les savoirs de référence. Cette rencontre se négocie à travers des actions diversifiées exercées par l'apprenant sur des formes de savoirs rendues manifestes et manipulables. La recherche sur les formes de savoirs conduit à varier les formes, à en analyser les atouts et les limites, et à ne pas privilégier seulement les formes discursives. Cette perspective permet de ne pas exclure les apprenants fragilisés par une maîtrise de l'écrit insuffisante : la présence de couleurs, de signes mathématiques et de photographies prises en contexte associées à une faible présence de l'écrit constituent autant d'éléments qui permettent la prise de repères pour les apprenants dont le parcours scolaire n'a pas permis la construction des savoirs dits « de base ». En rapportant les savoirs de référence au contexte particulier qui est le sien, l'apprenant peut travailler sur les affordances de son environnement, entendues comme relevant de la perception, par l'individu, de ce que l'environnement permet de réaliser au mieux (Gibson, 1977) [22]. L'hétérogénéité des personnes n'est pas pensée comme un problème, mais comme une richesse dont il est tenu compte dans la conception de situations qui pensent l'humain en tant qu'homme pluriel (Lahire, 1998) [32]. L'accent est mis sur la dimension analytique du travail à réaliser, le tout s'exerçant dans un espace confortable contextualisé (le nid) dans lequel l'apprenant est mis en condition de pouvoir travailler sur les savoirs en jeu avant de prendre les décisions qui seront les siennes, sans subordination à celles du professionnel éducateur. Les spécificités individuelles des individus sont intégrables sans exception : l'issue de chaque situation d'apprentissage se décline ainsi à travers une palette de solutions différentes et non pas à travers une seule. La construction de l'ensemble part de ce qu'est l'apprenant, le conduit à analyser les éléments de savoirs en jeu et lui permet ensuite de quitter la situation en ayant développé un travail cognitif et social personnalisé et contextualisé, en interaction avec les autres (Debussche \& Balcou-Debussche, 20II) [4I].

\section{La conception des dispositifs}

La conception de chaque dispositif d'apprentissage est réalisée à partir d'une double expertise : celle d'un professionnel de santé référent dans sa spécialité et celle d'une ethnosociologue de l'éducation et de la formation. Les deux domaines de compétences (les sciences médicales d'une part, les sciences humaines et sociales de l'autre) n'interviennent donc pas l'un après l'autre : c'est la co-construction de la situation d'apprentissage qui permet de s'assurer dès le départ que l'ensemble de la construction répond aux exigences des deux domaines. Tous les éléments constitutifs d'une situation d'apprentissage sont donc analysés finement par les deux concepteurs. En premier lieu, ils définissent un objectif général qui les conduit très vite à confronter leurs propres savoirs avec les savoirs actualisés de référence. Ce travail analytique leur permet de se départir de leurs propres représentations sur la question : la perspective générale est de réussir à mettre en jeu des éléments de savoirs actualisés, clairement identifiés et définis en référence aux savoirs savants disponibles. Les savoirs sont délimités en tenant compte des perspectives générales de l'action éducative et les liens entre les éléments de savoirs sont mis en évidence. La distinction est 


\section{L'éducation thérapeutique : entre savoirs complexes, formateurs, apprenants hétérogènes et contextes pluriels}

faite entre les concepts (les élaborations théoriques construites à partir des savoirs scientifiques), les opérateurs (ce qui permet d'agir sur les concepts) et les règles, recommandations et principes qui sont fonction des espaces temporels, culturels et sociaux concernés.

Dans un second temps, des rencontres et échanges permettent de recueillir des données sur les représentations des apprenants ainsi que sur les dimensions contextuelles dans lesquelles les individus s'inscrivent. Cette étape permet de prendre la mesure des représentations sociales, de mettre en évidence des priorités ainsi que des distances importantes entre les savoirs tels qu'ils sont mobilisés par les individus et les savoirs de référence. En tenant compte des résultats de l'analyse des savoirs et des représentations des apprenants, les experts procèdent à un ajustement qui tient compte de l'objectif général qui avait été fixé et de la faisabilité potentielle du travail éducatif. Dans un troisième temps, les concepteurs de la situation élaborent le dispositif d'apprentissage en tenant compte des éléments de connaissance dont ils disposent. Le travail sur l'agencement de la situation d'apprentissage se construit autour des points suivants : les résultats attendus, les formes de savoirs, les activités des apprenants, les orientations et les consignes, le rôle du formateur, les indicateurs de résultats et les régulations à prévoir. Enfin, dans un quatrième temps, la situation d'apprentissage est mise à l'épreuve du terrain. Des observations régulières sont conduites en situation de façon à percevoir les ajustements nécessaires à une meilleure construction des connaissances et des compétences psychosociales. Au bout de plusieurs expérimentations avec des personnes hétérogènes et dans des contextes différents, la situation d'apprentissage fait l'objet de régulations et d'ajustements qui portent sur les éléments contextuels, l'accès aux savoirs ou l'actualisation des données scientifiques.

\section{La mise en œuvre sur le terrain}

Les situations sont mises en place sur les différents terrains après une session de formation d'une à deux journées pour différents professionnels (médecins, infirmiers, pharmaciens, podologues, éducateurs en santé, enseignants, pairs éducateurs), sur chacun des thèmes abordés : la santé des artères, la maîtrise des graisses dans l'alimentation, la gestion de l'activité physique, la compréhension du diabète et de ses traitements, la compréhension des enjeux de la mise sous insuline. Au cours de chaque session, les professionnels bénéficient d'une actualisation des savoirs médicaux sur la question abordée, d'un cadrage théorique et pratique réalisé par l'expert en sciences de l'éducation et d'une réflexion sur la façon d'opérationnaliser la situation d'apprentissage avec un groupe d'une dizaine d'apprenants (Balcou-Debussche, 20II) [42]. De façon graduelle et progressive, le travail permet d'identifier clairement les objectifs de la situation, le déroulement dans ses grandes étapes, le matériel utilisé, les consignes à donner, les régulations à prévoir, les éléments de synthèse à analyser et à transmettre éventuellement à la structure coordinatrice (réseau, service hospitalier, association). Le formateur met ensuite la situation d'apprentissage en place pour des apprenants qui vont réussir à travailler à partir de leurs propres données, quelles que soient leurs particularités culturelles, religieuses ou socioéconomiques.

La situation éducative se démarque ainsi de la seule perspective qui consisterait à apprendre ce qu'il faut faire (conseils) et ce qu'il ne faut pas faire (interdits). Les différences de pratiques sont légitimées et respectées dès le départ. Le formateur laisse du temps à l'apprenant pour qu'il puisse formaliser les prises de conscience qui s'opèrent grâce à l'activité en situation. Les apprenants empruntent au monde scientifique ses modalités de travail : ils observent, émettent des hypothèses comparent, déduisent, mettent en relation. Ils analysent les bénéfices d'actions potentiellement réalisables dans des contextes ordinaires, puis ils en choisissent une ou deux, en tenant compte du bénéfice sur la santé et de la faisabilité de l'action en contexte, en continuité. Grâce à des analyses plurielles, l'apprenant quitte ainsi la situation d'apprentissage en ayant trouvé lui-même la (les) pratique (s) qui tient (tiennent) compte des contraintes et des atouts de sa vie sociale, familiale, personnelle. Dans les livrets de la collection Education et prévention des maladies chroniques (EPMC) ${ }^{1}$ qui servent de support à cette élaboration, les photographies prises dans différentes situations attestent de la prise en compte effective du contexte de l'apprenant, en même temps qu'elles le légitiment. La compétence de l'apprenant consiste alors à réaliser un objectif en utilisant les ressources de son environnement et en sachant choisir dans l'environnement les éléments qui présentent les atouts les plus favorables par rapport à l'action prévue. L'activité de l'apprenant est permanente, car la situation est pensée d'emblée pour que les actions exercées par chacun conduisent vers des élaborations cognitives dans lesquelles la dimension sociale est travaillée. La construction générale des situations d'apprentissage aboutit ainsi à une prise de décision négociée et réfléchie, quels que soient les apprenants. Dans la situation sur la santé des artères, l'apprenant se rend compte qu'il ne peut rien changer à certaines variables (l'âge, le sexe), mais qu'à l'inverse, il peut agir sur d'autres : la tension artérielle, le cholestérol, les triglycérides, la glycémie, le tour de taille, le tabac. Il évalue la santé de ses artères à partir de ses données personnelles et prend conscience de la possibilité d'augmenter son « total santé ». En mettant en relation ce total avec l'évolution lente, discrète et non douloureuse de la plaque d'athérome, l'apprenant prend conscience luimême de l'écart qui sépare son résultat avec le total santé maximal. Grâce à l'expérimentation, il prend aussi et surtout conscience de la possibilité d'augmenter son total en exerçant des actions spécifiques (marcher, manger équilibré, diminuer

${ }^{1}$ La collection EPMC est publiée aux Editions des Archives Contemporaines, à Paris. Voir dans Catalogues, Programme EPMC : http://www.archivescontemporaines.com/ 


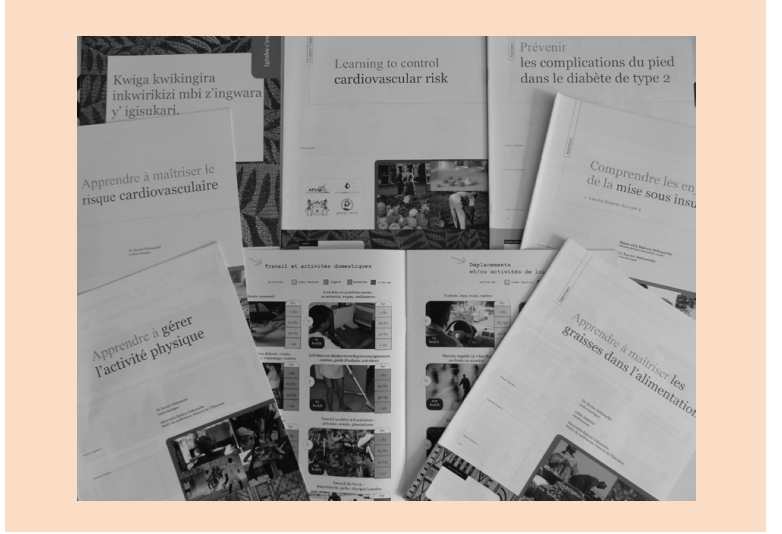

Illustration 1 : Les livrets de la collection Education et prévention des maladies chroniques

les grignotages). Après avoir identifié les bénéfices de chaque action, l'apprenant en choisit une ou plusieurs, en réfléchissant à la pertinence, à la faisabilité et à l'attractivité de chacune d'elles. Invitée à tenir compte de ses dispositions personnelles spécifiques et du contexte social et économique qui est le sien, la personne malade chronique peut alors s'engager à mener ou non l'action qu'elle a choisie.

Dans chaque situation d'apprentissage, des indicateurs de résultats déterminés à l'avance et formalisés dans le livret renseignent le formateur sur l'avancement des apprenants. Le formateur, mais aussi et surtout l'apprenant sont en mesure d'identifier précisément les points sur lesquels l'apprenant a travaillé, de mettre ses résultats en relation avec ceux des autres et d'identifier l'action (et les modalités) qu'il s'engage à mener. Le formateur dispose des mêmes indicateurs que l'apprenant, ce qui lui permet de savoir si chacun a compris ce qu'il y avait à comprendre.
Dans toutes les structures, les mêmes indicateurs sont relevés et soumis a minima à un comptage numérique, ce qui permet des évaluations à plus large échelle, sur un nombre important d'apprenants ainsi que sur des temps longs. En tenant compte des résultats obtenus et des progressions observées ou non, le formateur peut ainsi venir en aide aux apprenants qui en ont le plus besoin, à différentes étapes du processus. Cette construction vaut pour les trois thématiques présentées ci-après.

\section{Les analyses dans les réseaux de santé}

Dans le réseau Réunion Cœur Artères Rein Education (RéuCARE), premier terrain d'expérimentation, une analyse a porté sur les données récoltées auprès de 190 professionnels de santé et de 1317 apprenants inclus depuis le démarrage du réseau (décembre 2003) jusqu'en 2006. Parmi les professionnels, 41 d'entre eux ont été interviewés. 20 entretiens ont été réalisés avec des apprenants inclus depuis au moins deux ans (quinze d'entre eux avaient eu trois séances éducatives). Sur le plan médical, les données suivantes ont été récoltées : tension, glycémie ou hémoglobine glyquée, Low Density Lipoprotein (LDL) - cholestérol, High Density Lipoprotein (HDL) cholestérol, tour de taille, indice de masse corporelle. Une seconde analyse a pris en compte les indicateurs statistiques suivants : nombre d'inclusions, de formations, de séances d'éducation et de professionnels signataires du réseau. 25 I apprenants ayant bénéficié du cycle complet d'éducation (trois séances) ont répondu à un questionnaire décomposé en quatre parties : la satisfaction des personnes, le retentissement des activités du réseau, les pratiques de santé, les données sociodémographiques. 101 professionnels du réseau ont été interrogés sur la formation professionnelle, la communication avec le réseau, l'inclusion et le suivi des personnes, les séances d'éducation et la satisfaction globale.

\begin{tabular}{|l|l|l|l|}
\hline \multicolumn{4}{|c|}{ Séance thématique } \\
\hline & \multicolumn{1}{|c|}{ Santé des artères } & \multicolumn{1}{|c|}{ Apport des graisses } & \multicolumn{1}{c|}{ Activité physique } \\
\hline Identification des pratiques & $\begin{array}{l}\text { L'apprenant calcule son total } \\
\text { santé en tenant compte de } \\
\text { ses résultats biologiques (6 } \\
\text { variables) }\end{array}$ & $\begin{array}{l}\text { L'apprenant établit la liste des } \\
\text { aliments qu'il a consommés } \\
\text { dans une journée }\end{array}$ & $\begin{array}{l}\text { L'apprenant liste toutes les } \\
\text { activités menées dans une } \\
\text { semaine }\end{array}$ \\
\hline $\begin{array}{l}\text { Confrontation avec les savoirs } \\
\text { de référence }\end{array}$ & $\begin{array}{l}\text { L'apprenant met son total } \\
\text { santé en relation avec } \\
\text { l'évolution de la plaque } \\
\text { d'athérome }\end{array}$ & $\begin{array}{l}\text { L'apprenant met son total de } \\
\text { graisses consommées avec les } \\
\text { recommandations sur l'équilibre } \\
\text { alimentaire }\end{array}$ & $\begin{array}{l}\text { L'apprenant met son total } \\
\text { semaine en relation avec les } \\
\text { recommandations actuelles }\end{array}$ \\
\hline $\begin{array}{l}\text { Identifications d'actions à } \\
\text { mener }\end{array}$ & $\begin{array}{l}\text { L'apprenant découvre des } \\
\text { actions simples qui vont avoir } \\
\text { un bénéfice direct sur l'une ou } \\
\text { plusieurs des 6 variables }\end{array}$ & $\begin{array}{l}\text { L'apprenant découvre, pour } \\
\text { chaque aliment (I20 aliments } \\
\text { analysés) l'apport en graisses } \\
\text { saturées et insaturées }\end{array}$ & $\begin{array}{l}\text { L'apprenant découvre, pour } \\
\text { chaque activité, l'intensité et les } \\
\text { calories dépensées }\end{array}$ \\
\hline Analyse du contexte & $\begin{array}{l}\text { L'apprenant analyse son } \\
\text { environnement géographique, } \\
\text { humain ainsi que le coût } \\
\text { potentiel engendré par la mise } \\
\text { en place d'une nouvelle activité }\end{array}$ & $\begin{array}{l}\text { L'apprenant analyse ses modes } \\
\text { d'approvisionnement et de } \\
\text { stockage des aliments, ses } \\
\text { façons de cuisiner et de manger }\end{array}$ & $\begin{array}{l}\text { L'apprenant analyse son } \\
\text { environnement géographique, } \\
\text { humain ainsi que le coût } \\
\text { engendré par la mise en place } \\
\text { d'une nouvelle activité }\end{array}$ \\
\hline
\end{tabular}

Tableau 1

Activités réalisées en situation par l'apprenant et principaux indicateurs, aux différentes étapes.

52 • Recherche en soins infirmiers $n^{\circ} 110$ - Septembre 2012 Copyright $\odot$ ARSI tous droits réservés 


\section{L'éducation thérapeutique : entre savoirs complexes, formateurs, apprenants hétérogènes et contextes pluriels}

Dans les autres contextes (île Maurice, Burundi, Botswana, Mali, Guyane, Mayotte), les analyses ne sont pas aussi complètes du fait d'un manque de ressources humaines pour les données de la recherche, la priorité allant le plus souvent au développement du travail éducatif en direction des personnes malades. Cependant, le nombre de séances réalisées par les professionnels formés et dans les différentes structures est connu, ainsi que les indicateurs relevés en séance tels que les variables agissant sur la santé des artères : index de masse corporelle, glycémie, tension artérielle, tabac, actions décidées par les apprenants. Toutes les fois que cela a été possible, l'analyse quantitative s'est appuyée sur les calculs de fréquences et de pourcentages avec une utilisation du test du chi-2 pour comparer les données entre les différentes structures. Elle a été complétée par une étude de la moyenne et de l'écart-type pour les variables quantitatives clinicobiologiques et par l'utilisation du test $t$ de Student pour les séries appariées, en comparaison avant/après des données. A partir de données qui portent tout autant sur les résultats obtenus par les apprenants au cours des situations d'apprentissage (bilans, analyses des pratiques ordinaires, choix d'actions) que sur les comptes-rendus réguliers établis par les équipes d'encadrement, le travail qui s'est exercé autour des nids d'apprentissage peut être présenté comme le montre le tableau 2 ci-dessous.

Chaque structure qui propose les situations d'apprentissage demande un accord préalable au participant. Après avoir reçu une information sur les modalités des séances éducatives qui vont lui être proposées, le participant peut accepter ou non de s'engager. Il conserve le droit d'interrompre le cycle éducatif à tout instant. La confidentialité des données est assurée par l'attribution d'un identifiant.

\section{RESULTATS OBTENUS}

\section{L'impact du cycle initial}

Entre 2004 et 2006, la plupart des professionnels de santé inclus dans le réseau RéuCARE ont mené au moins une situation d'apprentissage (6I \% d'entre eux) et $65 \%$ ont déclaré vouloir continuer. Le travail éducatif en réseau leur a permis d'améliorer leurs pratiques professionnelles en direction des personnes à risque ( $80 \%$ d'entre eux) ou en direction des autres (66\%). Les professionnels de santé parlent de véritable changement de leurs méthodes de travail, mais $68 \%$ d'entre eux soulignent aussi les changements des apprenants depuis leur prise en charge éducative. $95 \%$ des professionnels se disent satisfaits voire très satisfaits.

Durant les deux premières années (2004-2006), $25 \%$ des I 317 apprenants inclus dans le réseau RéuCARE ont bénéficié d'un cycle initial complet (santé des artères, alimentation, activité physique). Toutes les personnes qui ont bénéficié du cycle sont satisfaites ( $98 \%$, dont $86 \%$ très satisfaites). La satisfaction porte sur le contenu des séances, le choix des thèmes, le mode collectif des séances et des outils, les connaissances et les conseils apportés. Les personnes apprécient la démarche de choix d'actions pour la santé et la jugent très motivante. Plus de $90 \%$ déclarent que les situations leur ont permis de mieux comprendre la santé des artères et le problème des graisses dans l'alimentation. 91 \% des apprenants déclarent avoir changé leurs habitudes alimentaires après la séance. Les résultats cliniques et biologiques, avec un recul minimum de huit mois entre l'inclusion dans le réseau et le recueil des données chez les sujets ayant bénéficié d'un cycle complet montrent une évolution favorable : amélioration de I'hémoglobine glyquée $(7,81 \pm 1,50 \%$ versus $8, I I \pm 1,54 \%$, $\mathrm{p}<0,005$ ), amélioration des paramètres lipidiques (Low Density Lopoprotein cholestérol et High Density Lopoprotein cholestérol améliorés : respectivement $1,20 \pm 0,43$ versus $\mathrm{I}, 32 \pm 0,0,25 \mathrm{~g} / \mathrm{l}$ et $0,38 \pm 0, \mathrm{I}$ I g/l vs $0,35 \pm 0,12 \mathrm{~g} / \mathrm{l}, \mathrm{p}<0,005)$, de la pression artérielle $(138,4 \pm 15,5 \mathrm{vs} 143,0 \pm 14,6 \mathrm{mmHg}$, $\mathrm{p}<0,005)$ et du tour de taille $(108,4 \pm 7,6 \mathrm{~cm}$ vs $109,5 \pm$ $7,5 \mathrm{~cm}, \mathrm{p}<0,05)$. Les résultats obtenus dans les différents lieux montrent que les actions choisies dépendent non seulement des individus, mais aussi des contextes (cf tableau 3).

\begin{tabular}{|l|l|l|l|l|l|}
\hline \multicolumn{1}{|c|}{ Lontexte } & \multicolumn{2}{c|}{ Thématique } \\
\hline \multicolumn{1}{|c|}{$\begin{array}{c}\text { Cadre } \\
\text { institutionnel }\end{array}$} & \multicolumn{1}{c|}{ Public concerné } & Santé artères & \multicolumn{1}{c|}{$\begin{array}{c}\text { Apport des } \\
\text { graisses }\end{array}$} & \multicolumn{1}{c|}{$\begin{array}{c}\text { Gestion de } \\
\text { l'activité } \\
\text { physique }\end{array}$} \\
\hline $\begin{array}{l}\text { Île de la } \\
\text { Réunion }\end{array}$ & $\begin{array}{l}\text { Réseau de santé } \\
\text { RéuCARE }\end{array}$ & $\begin{array}{l}\text { Risque cardiovasculaire, } \\
\text { Diabète }\end{array}$ & $\begin{array}{l}841 \text { patients } \\
125 \text { séances }\end{array}$ & $\begin{array}{l}567 \text { patients } \\
92 \text { séances }\end{array}$ & $\begin{array}{l}327 \text { patients } \\
54 \text { séances }\end{array}$ \\
\hline $\begin{array}{l}\text { Île de la } \\
\text { Réunion }\end{array}$ & Éducation nationale & $\begin{array}{l}\text { Élèves de 4è et 3è de } \\
\text { collège }\end{array}$ & \multicolumn{1}{|c|}{$\begin{array}{l}157 \text { élèves } \\
4 \text { séances }\end{array}$} & - \\
\hline Île Maurice & $\begin{array}{l}\text { Réseau } \\
\text { communautaire }\end{array}$ & Risque cardiovasculaire & $\begin{array}{l}303 \text { patients } \\
74 \text { séances }\end{array}$ & $\begin{array}{l}120 \text { patients } \\
24 \text { séances }\end{array}$ & $\begin{array}{l}63 \text { patients } \\
14 \text { séances }\end{array}$ \\
\hline Burundi & $\begin{array}{l}\text { Réseau de santé } \\
\text { communautaire }\end{array}$ & Risque cardiovasculaire & $\begin{array}{l}1970 \text { patients } \\
218 \text { séances }\end{array}$ & $\begin{array}{l}287 \text { patients } \\
41 \text { séances }\end{array}$ & - \\
\hline
\end{tabular}

Tableau 2

Mise en place des situations d'apprentissage dans des contextes culturels et institutionnels différents (2007-2009). 


\begin{tabular}{|l|c|c|c|c|}
\cline { 2 - 5 } \multicolumn{1}{c|}{} & $\begin{array}{c}\text { RéuCARE } \\
(\mathbf{n = 8 4 I )}\end{array}$ & $\begin{array}{c}\text { CHU } \\
(\mathbf{n = 2} / \mathbf{0})\end{array}$ & $\begin{array}{c}\text { Maurice } \\
(\mathbf{n = 8} / 5)\end{array}$ & $\begin{array}{c}\text { Burundi } \\
(\mathbf{n}=\mathbf{1 2} \text { II) }\end{array}$ \\
\hline Dates & $\begin{array}{c}05 / 2007- \\
05 / 2009\end{array}$ & $\begin{array}{c}06 / 2008- \\
05 / 2009\end{array}$ & $\begin{array}{c}06 / 2007- \\
06 / 2009\end{array}$ & $\begin{array}{c}08 / 2007- \\
12 / 2008\end{array}$ \\
\hline Nombre d'action(s) par participant & $0-3$ & $1-3$ & $0-3$ & $1-4$ \\
\hline
\end{tabular}

Actions choisies (\%)

\begin{tabular}{|l|c|c|c|c|}
\hline Je mange moins salé & 7,6 & 6,0 & 7,9 & $27,5 *$ \\
\hline Je mange des légumes et un fruit à chaque repas & 12,7 & 15,7 & 16,7 & $9,8 \S$ \\
\hline Je diminue les sucreries et j'arrête le grignotage & 10,8 & $20,9 \S=$ & 14,8 & $19,7 *=$ \\
\hline Je prends des repas équilibrés & 10,5 & $14,8 \S$ & 9,1 & $4,8 *$ \\
\hline Je limite l'apport de graisses & $17,2 \S$ & $28,6 *$ & 13,7 & $41,0 *$ \\
\hline Je me limite à un verre d'alcool/jour & 1,1 & 2,9 & 2,0 & $7,3 \$$ \\
\hline Je marche $30 \mathrm{mn} /$ jour & 18,8 & $29,5 \$$ & 19,5 & $30,6 \$$ \\
\hline Je marche $30 \mathrm{mn} 3$ fois par semaine (ou équivalent) & 10,3 & 14,3 & 9,4 & 10,6 \\
\hline Je prends régulièrement mes médicaments & 2,1 & 4,3 & 5,9 & $8,5=$ \\
\hline J'arrête de fumer & 2,3 & 4,8 & 1,0 & $7,2 \$$ \\
\hline
\end{tabular}

$* p<0,001$ vs les 3 autres lieux $\$ p<0,001$ vs Maurice et RéuCARE $\quad \S p<0,05$ vs Maurice $=p<0,001$ vs RéuCARE (test du Chi-2)

Tableau 3

Actions choisies par les apprenants

\section{Un accès possible à un grand nombre}

Depuis 2004, les situations d'apprentissage sont proposées à des apprenants très différents, dont des adultes et des adolescents présentant des parcours de socialisations fort divers, notamment d'un point de vue familial, scolaire ou professionnel. Les situations sont mises en œuvre dans des contextes tout aussi différents qui vont de l'île de la Réunion à l'île Maurice et au Burundi, en passant par la France métropolitaine, la Guyane, le Botswana, Mayotte et le Mali. Toute personne qui intègre la structure ou l'institution qui diffuse l'approche (l'hôpital, le réseau de santé, le réseau communautaire, l'association) se voit proposer l'une ou l'autre des situations d'apprentissage, ou les trois : apprendre à maîtriser la santé des artères, apprendre à maîtriser l'apport des graisses dans l'alimentation, apprendre à gérer son activité physique ordinaire. Selon les cas, d'autres thématiques peuvent être proposées en premier lieu ou en complément du cycle initial, comme par exemple comprendre le diabète et ses traitements, ou comprendre les enjeux de la mise sous insuline.

Durant les cinq premières années du développement de l'approche, plus de 3000 apprenants ont bénéficié d'une, deux ou trois situations d'apprentissage. Depuis 2004, ce sont au total plus de 10000 personnes malades chroniques qui ont bénéficié d'un accompagnement éducatif reposant sur l'approche intégrative proposée dans les situations d'apprentissage.

\section{Des formateurs soignants et non soignants}

Cet important développement a été rendu possible par le fait que les nids d'apprentissage apparaissent sous forme de publications complètes qui comprennent un guide pédagogique détaillé à destination du formateur, ainsi que des livrets en couleurs que les apprenants emportent chez eux. Adaptés aux contextes dans lesquels ils sont utilisés, les livrets sont désormais traduits dans les langues des pays concernés : en kirundi pour le Burundi, en anglais et en setswana pour le Botswana, pays qui travaille depuis deux ans sur le développement des nids d'apprentissage à l'échelle nationale. Au Botswana, un travail de collaboration étroite a été effectué avec le Ministère de la santé du pays et l'Association pour la promotion de la santé internationale (APSA) Cette Organisation non gouvernementale (ONG) a été à l'initiative des développements réalisés à l'île Maurice et au Burundi, un important projet de développement ayant été soutenu par la World Diabetes Foundation (WDF). Au Mali, le partenariat de recherche et de développement s'est concrétisé grâce 


\section{L'éducation thérapeutique : entre savoirs complexes, formateurs, apprenants hétérogènes et contextes pluriels}

à l'ONG Santé Diabète, avec la mise en place de cycles d'éducation de groupe par des pairs éducateurs formés aux situations d'apprentissage. En France métropolitaine, ce sont surtout les maisons du diabète et la maison Diabète-ObésitéRisque Cardiovasculaire (DOC) de la Mutuelle générale de l'éducation nationale (MGEN) qui relaient le travail et proposent les situations d'apprentissage aux personnes obèses, diabétiques et/ou à risque cardiovasculaire. $\mathrm{A}$ Mayotte, un partenariat de recherche et de développement s'est concrétisé avec le Réseau Diabète Mayotte (REDIABYLANG), le projet de recherche présenté par l'Université de La Réunion ayant été validé et soutenu par le Ministère de l'outre-mer. Dans les différents pays, l'accompagnement a été réalisé par des acteurs différents (médecins, infirmiers, aides-soignants), mais aussi par des patients experts qui ont pu accompagner un processus qui présente un certain nombre de garanties qu'il est important de pointer ici.

La première est la double validation du dispositif, dès le départ, par des experts des sciences médicales et des experts des sciences humaines et sociales. C'est bien parce qu'un travail conjoint a fait l'objet d'analyses approfondies qui se sont inscrites sur environ cinq années d'expérimentations et de régulations que le processus peut ensuite s'autonomiser et devenir accessible à d'autres acteurs qui peuvent s'en faire le relais. L'une des caractéristiques principales de la collection EPMC est précisément de pouvoir faire l'objet de régulations et d'actualisations permanentes, notamment par l'expert médical qui travaille au plus près des savoirs scientifiques disponibles sur la thématique traitée. En suivant l'agencement du dispositif et en respectant son contenu, l'éducateur est en condition de ne pas faire d'erreurs majeures puisque l'ensemble des analyses a donné lieu à une double validation (sciences médicales et sciences sociales) complétée d'expérimentations dans divers lieux. Si l'éducateur doit se porter garant du bon déroulement de la situation d'apprentissage et de la dynamique qu'il est nécessaire de créer, les experts des sciences médicales et des sciences humaines et sociales se portent garants de la validité et de l'actualité des savoirs mis en jeu dans le dispositif, ainsi que de la pertinence et de l'adéquation de l'agencement général proposé.

La seconde particularité est liée à un cadrage précis qui propose une conduite de la séance étape par étape, l'agencement général obéissant à des conditions telles qu'elles présentent un maximum de garanties de pouvoir obtenir des résultats avec les apprenants. La sélection de formes de savoirs adaptées et pertinentes, et le choix d'activités variées sont autant d'éléments qui maintiennent en permanence l'intérêt de l'apprenant. Pour le formateur, les consignes sont claires (elles sont écrites dans le livret du formateur) et les indicateurs de réussite sont clairement identifiés. Les apprenants gardent la possibilité, sur le long terme, de se référer à des savoirs de référence qu'ils comprennent puisqu'ils partent chez eux en emportant un support qui a été travaillé en situation avec l'accompagnement du formateur.
Tout au long de la situation d'apprentissage, le formateur peut ainsi s'assurer que l'apprenant comprend ce qui est à comprendre : lorsque l'apprenant quitte la situation éducative, le formateur est assuré de la compréhension et de la réception que l'apprenant a pu réaliser durant la séance. Une telle situation n'a rien à voir avec la distribution de documents dits « pédagogiques » dont on ne contrôle en rien la réception puisque cette dernière est le plus souvent livrée à la seule discrétion de celui à qui l'on remet le document. Par ailleurs, dans les deux livrets (formateur et apprenant), l'inscription des résultats sur un temps long est visualisée par la présence d'un tableau synthétique qui organise le suivi sur cinq ans : une personne confrontée à des problèmes de surpoids, d'obésité ou de pathologie cardiovasculaire voit ainsi que les résultats s'obtiennent non pas sur quelques jours, mais sur plusieurs mois. Enfin, toutes les fois que c'est nécessaire, la nécessité de solliciter ou consulter le corps médical est signalée dans le livret de l'apprenant (et bien sûr, dans le livret du formateur), ce qui permet au formateur de ne prendre aucun risque pour la santé de la personne.

La troisième condition de développement réside dans la formation des acteurs, dont les pairs éducateurs ou les patients experts. Pour réussir à respecter le cadrage fixé et ne pas aller au-delà de son rôle d'éducateur et/ou d'accompagnateur en éducation thérapeutique, des temps d'appropriation du dispositif et de réflexion sont programmés d'emblée car ils sont considérés comme étant absolument nécessaires. Le cadrage comprend plusieurs volets. En premier lieu, la clarification du positionnement de l'éducateur et/ou accompagnateur en éducation thérapeutique par rapport au monde soignant. Lors de la formation, les formateurs de formateurs précisent notamment que l'éducateur et/ou l'accompagnateur en éducation thérapeutique n'est ni médecin, ni infirmier, ni pharmacien, ni professeur dans un champ disciplinaire précis. Cette clarification a le mérite de poser les limites de l'action possible de chaque professionnel en même temps qu'elle permet d'emblée de penser la nécessaire complémentarité des actions des uns et des autres. En second lieu, le cadrage fait l'objet d'une sélection des pairs éducateurs et d'un suivi régulier de leur travail. Ainsi, au Mali, chaque pair éducateur a d'abord reçu une formation aux « nids d'apprentissage » dans le cadre d'une rencontre de trois jours (première année) suivie d'un complément de trois autres journées (seconde année). Au cours de chaque temps de formation, les candidats pairs éducateurs ont eu l'occasion de s'approprier les supports utilisés, d'analyser les contenus proposés et de se mettre peu à peu en perspective de pouvoir assurer le travail d'accompagnement du dispositif, en vue de son extension et de son développement. Fort de cet ensemble de clarifications, chaque candidat a bien conscience que tout manquement peut faire l'objet d'une non sélection dans le groupe de formateurs. Les candidats sont mis en situation de pratiquer à leur tour en adoptant la posture, non plus de l'apprenant, mais celle du formateur. A l'issue de ce processus de formation, les personnes qui restent volontaires et sont jugées capables 
d'assurer l'accompagnement de l'approche éducative sont amenées à exercer le travail avec de nouvelles personnes malades chroniques. Un processus de suivi est mis en place à travers un travail d'analyse continu qui conduit à voir les formateurs en situation et à s'assurer du non dépassement des fonctions et des rôles assignés (Debussche \& al., 2010) [43].

\section{DISCUSSION}

\section{Des atouts, mais aussi des limites}

La construction des situations d'apprentissage telles qu'elles ont été expérimentées depuis maintenant presque une dizaine d'années apparaît féconde, tant pour les perspectives que ces orientations offrent pour le développement de l'action que pour leur intérêt en matière de recherche. Les résultats obtenus sont à mettre en relation avec des actions d'éducation de groupe dont l'utilité et les effets positifs ont été soulignés par ailleurs dans les maladies chroniques (Lorig \& al., 200I) [44] ; (Norris et al., 200I) [45]. Les stratégies mobilisées renvoient aux approches cognitives et comportementales en associant la résolution de problème et en tenant compte des contextes individuels et sociaux. Elles permettent la fixation d'objectifs réalistes et réalisables : préparation et capacité au changement, centration sur la personne, apprentissages sur les compétences et les capacités d'adaptation (Peyrot et Rubin, 2007) [46]. Elles travaillent aussi sur la nécessité d'agir en proximité et sur un plan communautaire, en prenant en compte le contexte de l'individu (Hawthorne et al., 2008) [47]. D'ordinaire, dans la relation triangulaire qui met en jeu le formateur, les savoirs et l'apprenant, le formateur reste le plus souvent maître des savoirs sans que ces derniers soient directement accessibles pour les apprenants. Le formateur s'octroie souvent le droit de pouvoir négocier et de donner des conseils en fonction de ce qu'il pense être bon pour l'apprenant, en référence à des recommandations standardisées établies à partir de profils d'individus standard, mais aussi en référence à des valeurs que le formateur peut penser comme étant universellement partagées. Un tel cadre d'action est souvent opératoire pour des situations dans lesquelles l'expertise du formateur doit permettre de répondre rapidement à une situation donnée (face à une blessure, par exemple), mais il ne l'est pas forcément dans le cadre d'une gestion ordinaire de la maladie chronique qui exige de l'apprenant qu'il agisse au quotidien sur un temps long, en tenant compte de sa culture, de ses priorités, de ses valeurs et des contextes sociaux dans lesquels il évolue. En travaillant sur la perspective d'une appropriation effective des savoirs par les apprenants et en intégrant le travail dans la durée, en lien avec le temps et les espaces qu'ils traversent, les situations d'apprentissage analysées ici supposent que le formateur accepte l'idée d'une appropriation effective des savoirs par l'apprenant, ce qui va de pair avec l'acceptation de rapports de pouvoir différents de ce qu'ils sont souvent. Or, cette question n'est pas sans poser un certain nombre de problèmes qui relèvent tout autant des formateurs et des apprenants eux-mêmes que des héritages organisationnels dans lesquels les actions doivent s'inscrire. L'une des principales difficultés est liée au formateur à qui l'on ne demande surtout pas d'être un diagnostiqueur ou un prescripteur. La mise en place des nids d'apprentissage questionne nécessairement les identités professionnelles, notamment celles de soignants qui ont un travail important à faire pour devenir formateurs et/ou éducateurs. Dans la situation d'apprentissage, le formateur n'utilise pas le discours pour expliquer les phénomènes que les apprenants vont être amenés à comprendre, pas plus qu'il ne l'utilise pour convaincre ou faire prendre une décision à tout prix. Le formateur prépare l'agencement matériel de la situation, donne les orientations et les consignes, aide l'apprenant à élaborer les connaissances, observe ce que chacun réalise et écoute ce que chacun dit. II éduque la capacité d'attention de l'apprenant et aide ceux qui éprouvent des difficultés particulières, notamment par rapport à la maîtrise de la langue française, de la langue écrite ou des relations sociales. Le formateur stimule et soutient l'activité et surveille les indicateurs de résultats. Dans le monde des soignants, cette posture qu'il doit adopter afin de laisser l'entière possibilité de réalisation à l'apprenant n'est pas conforme à ce qu'il a appris à faire avec des personnes qui restent le plus souvent pensées autour du modèle idéal (Hughes, 2003) [3I].

Les difficultés ne sont pas moindres lorsque l'on analyse ce qui se passe du côté des apprenants eux-mêmes. L'individu qui se voit proposer une situation d'apprentissage ne saisit pas forcément d'emblée ce qui est attendu de lui. Les représentations sociales liées à la santé et/ou à la maladie sont prégnantes (Herzlich, 1986) [9] ; (Laplantine, 1993) [10]. En conséquence, l'apprenant attend souvent du professionnel expert qu'il lui dicte ce qu'il a à faire et qu'il lui prescrive les actions à mener comme il se ferait prescrire des médicaments (Gravois Lee et Garvin, 2003) [28]. Dans les cadres spécifiques de l'éducation à la santé et de l'éducation thérapeutique, éduquer c'est aider l'apprenant à participer activement à la gestion de sa santé et il faut souvent du temps pour que se mettent en place des pratiques auxquelles certains ne sont pas habitués, comme par exemple venir dans la séance éducative avec les résultats antérieurs, ou choisir une action qui soit différente de celle de son voisin. II apparaît ainsi que si la dimension micro des situations d'apprentissage est désormais maîtrisée et opérationnelle, les dimensions meso et macro contextuelles sont encore à travailler afin d'autoriser des extensions qui pourraient largement dépasser ce qui est obtenu à l'heure actuelle.

\section{Des développements à poursuivre}

La conception et le développement de dispositifs d'apprentissage apparaît néanmoins comme l'une des illustrations possibles des voies concrètes à travers lesquelles la recherche en sciences 


\section{L'éducation thérapeutique : entre savoirs complexes, formateurs, apprenants hétérogènes et contextes pluriels}

humaines et sociales, en collaboration avec les sciences médicales, peut contribuer utilement à la réflexion et à l'action publique sans affaiblir le travail scientifique qui constitue sa spécificité. Cette co-construction se heurte cependant à l'inertie des institutions et aux socialisations professionnelles des soignants qui ne vont pas nécessairement dans ce sens (Balcou-Debussche, 2003) [23] ; (Balcou-Debussche, 2004) [24]. Les formateurs doivent encore apprendre à partir des interprétations différenciées de la santé et de la maladie dans des environnements tout aussi différenciés plutôt que de continuer à évoquer les actions et les savoirs des individus en termes de manques de connaissances, de motivation, de moyens ou d'incompétences (Balcou-Debussche, 2006) [14]. II s'agit là d'un travail difficile qui nécessite une expertise et du temps, et il n'est pas certain qu'actuellement les professionnels formés dans des logiques strictement disciplinaires construites autour d'un découpage par pathologies soient les mieux placés pour le faire (Balcou-Debussche, Debussche, 2008) [30], notamment si l'on considère que l'accès à l'éducation pour un plus grand nombre reste un obstacle important dans la maladie chronique et l'éducation en santé (Peyrot et al., 2009) [46].

Dans les pays dits développés, les résultats montrent que la mise en place de telles dynamiques constitue une complémentarité intéressante au suivi médical, en parallèle à l'expertise diagnostique telle qu'elle est pratiquée actuellement. Les situations d'apprentissage peuvent se décliner à travers des cycles éducatifs de groupe, mais elles peuvent aussi être mobilisées en tant que support individuel permettant de mieux articuler l'action des professionnels éducateurs avec celle des professionnels soignants. Actuellement, plusieurs expérimentations vont dans ce sens, avec notamment un travail en direction des médecins généralistes qui voient la possibilité d'inscrire le travail éducatif qu'ils revendiquent dans les cadres de fonctionnement qui sont les leurs. Les analyses et les expertises qui ont été réalisées autour des situations d'apprentissage permettent désormais de mieux penser leur inscription dans des organisations différentes de celles dans lesquelles elles ont été pensées au départ. Les réflexions en cours concernent ainsi la possibilité de nouveaux découpages des situations afin de les intégrer dans le cadre de plusieurs consultations conçues comme une suite d'actions éducatives complémentaires et capables de produire, à terme, les résultats attendus en matière de gestion de la maladie chronique. Le travail d'objectivation qui a déjà été réalisé autour des appropriations des savoirs en jeu en lien avec les contextes, avec un ensemble d'indicateurs précis qui permettent une lisibilité de l'avancement particulier de chaque apprenant, peut en effet contribuer, à coût restreint, à un développement du travail éducatif par des acteurs différents, dans de nouvelles organisations et structures. De telles extensions devraient permettre une diminution de la charge économique et financière que les actions d'éducation et de prévention représentent, y compris parce qu'une évaluation précise des actions est possible et qu'en les analysant régulièrement, les indicateurs pourraient permettre de mieux esquisser les priorités pour les actions à venir, en suivant au plus près les évolutions des sociétés et les problèmes de santé publique qui s'y posent.

Dans les pays en développement, les situations d'apprentissage ont déjà montré leur potentiel d'inscription, ce qui n'empêche pas de voir apparaître d'importantes différences de résultats selon les différents pays. La fragilité politique ou économique des pays concernés, ou encore les priorités fort diverses des individus font que l'approche bénéficie d'une inscription sociale et culturelle plus ou moins réussie. Au Burundi, le programme éducatif n'a pas pu se poursuivre au-delà des trois années d'expérimentation faute de stabilité politique et de financements. Au Mali, les événements politiques récents ont fragilisé le développement d'un processus qui réussissait pourtant à s'inscrire dans des perspectives micro territoriales, impliquant ainsi des associations, des espaces communautaires et les structures hospitalières. Au Botswana, dans un cadre politique et économique beaucoup plus stable, le développement de l'approche s'organise progressivement à travers une collaboration étroite qui vise tout autant l'extension nationale du dispositif que la possibilité, pour les individus, de se reconnaître dans des façons de penser et de faire qui ne leur semblent pas étrangères. Le travail d'adaptation qui s'est engagé a pris du temps, mais c'est aussi le garant d'une inscription sociale et culturelle pérenne, en vue de résultats qui mériteront d'être analysés sur les court, moyen et long termes. Enfin, des analyses plus fines sont en cours à Mayotte où un projet de recherche a permis de récolter de nouvelles données auprès des personnes, par le biais d'entretiens qui sont en cours d'analyse. A La Réunion, l'analyse des liens entre les pratiques langagières et les appropriations de savoirs lors des situations d'apprentissage apporte des éclairages intéressants sur les possibilités de développer des compétences en littératie en santé (Balcou-Debussche, 20I2) [48]. L'impact d'un cycle éducatif sur les données biologiques de personnes diabétiques de type 2 est en cours (Debussche, Collin, Fianu \& al., 2012) [49], de même que la faisabilité de l'approche par des professionnels divers et formés à cet effet. Dans une perspective de développement des alliances pluridisciplinaires (Billon-Descarpentries, 2003) [50], le travail de recherche se poursuit également grâce à un renforcement important des ressources humaines du fait de partenariats qui s'établissent sur le plan régional entre I'Université et le Centre hospitalier universitaire $(\mathrm{CHU})$ de La Réunion, mais aussi sur le plan national et international avec l'inscription de ces travaux dans les développements scientifiques de l'Institut français d'éducation (IFé) et du réseau UNIRéS (UNIversités en Réseau pour l'éducation à la Santé).

\section{CONCLUSION}

Conçues à partir de travaux scientifiques issus des sciences médicales et des sciences humaines et sociales, les situations d'apprentissage analysées ici apportent une contribution à la réflexion et au développement des actions en éducation thérapeutique. Leur potentiel d'intégration dans des contextes 
différenciés est un atout ; les résultats encourageants obtenus dans différents espaces avec des apprenants de profils différents en sont un autre. En France, cet apport s'inscrit dans un cadre général où des efforts importants sont encore à faire en termes de professionnalisation des formateurs en éducation thérapeutique et des apprenants eux-mêmes, tant du côté de la dimension épistémologique que praxéologique. Le fort développement des maladies chroniques, l'âge de plus en plus avancé des personnes concernées, la pénurie de médecins spécialistes dans certaines spécialités et dans certains secteurs risquent de conduire vers des mutations importantes du côté des pratiques éducatives. Dans les années à venir, les évolutions sont donc à analyser au fil des possibilités qui sont offertes, notamment dans les sphères où les mutations plurielles et rapides autorisent des agencements particuliers du côté de la recherche et des possibilités d'action. En accueillant les situations d'apprentissage comme des expériences prometteuses pour l'éducation dans les structures de soins, de santé et de prévention, les espaces insulaires périphériques tels que La Réunion, Maurice ou Mayotte, et les pays en développement comme le Burundi ou le Mali constituent, de ce point de vue, des observatoires précieux que les recherches en cours continuent à explorer.

Il serait difficile de conclure sans souligner le fait que ce type de développement ouvre aussi et surtout un large champ d'exploration pour les recherches en soins infirmiers. Le travail à poursuivre est d'une telle ampleur qu'il laisse nécessairement une part importante à chaque acteur ou à chaque corps professionnel qui projette de s'en saisir. Dans une période où les dossiers relatifs à l'éducation thérapeutique sont en mouvement et où les positionnements des uns et des autres peuvent se négocier, le corps infirmier a beaucoup à apporter en prenant plus amplement la place qui lui revient. Dans les différentes structures et organisations en France, la profession infirmière est abondamment sollicitée pour le travail praxéologique à exercer autour des pratiques d'éducation thérapeutique, mais force est de constater qu'elle l'est beaucoup moins dans les instances décisionnelles et/ou scientifiques. II reste donc à penser la façon dont les différentes professions qui œuvrent pour le maintien de la santé des individus pourraient construire ensemble des agencements cohérents capables de garantir aux malades chroniques un accès équitable et performant à la prévention, à l'éducation et aux soins. Face à ce vaste programme, le corps infirmier ne peut qu'interroger les voies par lesquelles de nouveaux espaces de réalisation, d'émancipation et de reconnaissance pourraient se révéler et s'affirmer. Plusieurs chantiers sont encore à ouvrir en France et en dehors, tant en explorant le développement de nouvelles dynamiques humaines, sociales et organisationnelles sur différents terrains qu'en construisant un projet scientifique solide, capable d'apporter les éclairages nécessaires à une compréhension plus fine de la complexité de l'éducation thérapeutique et de la diversité des éléments en jeu.

\section{Références bibliographiques}

1. DECCACHE A. Teaching, training or educating patients ? Influence of contexts and models of education and care on practice in patient education. Patient Education and Counselling ; 1995 ; 26 : 119-29.

2. MANDERSCHIED JC. Quelles recherches pour l'éducation à la santé ? Revue française de pédagogie ; 2006 ; 114 : 53-65.

3. FOUCAUD J, BURY J, BALCOU-DEBUSSCHE J, EYMARD C. Éducation thérapeutique du patient. Modèles, pratiques et évaluation. Saint-Denis : éditions INPES ; 2010.

4. BILLON J. Essai de théorisation des modèles de l'éducation dans le champ de la santé. Spirale ; 2000 ; 25 : 17-30.

5. SKINNER BF. Science and human behavior. New York Macmillan ; 1953.

6. PIAGET J. Théorie du langage, théorie de l'apprentissage. Paris Seuil ; 1979.

7. VYGOTSKI LS. Mind and society : the development of higher psychological processes. Cambrige MA : Harvard University Press ; 1978.

8. ROGER M. Les actions didactiques : principes et réalisations. In : École et Éducation, TUPIN F. (sous la direction de). Paris Anthropos-Economica ; 2003.

9. HERZLICH C. Représentations sociales de la santé et de la maladie et leur dynamique dans le champ social. In : L'étude des représentations sociales, DOISE W \& PALMONARI A. (sous la direction de). Genève Delachaux et Niestlé ; 1986.

10. LAPLANTINE F. Anthropologie de la maladie. Paris PayotRivages ; 1993.

11. LAWTON J. Lay experiences of health and illness : past research and future agendas. Sociology of Health \& IIIness; 2003 ; 25(3) : 23-40.

12. WOLFF E \& WATIN M. La Réunion : une société en mutation. Paris : Anthropos-Économica. Collection Univers créoles 7 ; 2010.

13. BALCOU-DEBUSSCHE M. Manger et se soigner : entre manques et excès. In : La Réunion : une société en mutation, WOLFF E \& WATIN M. (sous la direction de). Paris : Anthropos-Économica. Collection Univers créoles $7 ; 2010$.

14. BALCOU-DEBUSSCHE M. L'éducation des maladies chroniques. Une approche ethnosociologique. Paris : Éditions des archives contemporaines ; 2006.

15. DEBUSSCHE X, ROLLOT O, LE POMMELET C, FIANU A, LE MOULLEC N, REGNIER C, BOYER MC, COGNE M, BAKIRI F, SCHWAGER JC, FAVIER F. Quarterly and individual outpatient lifestyle counseling after initial inpatient education in type 2 diabetes : the REDIA-prev2 randomized controlled trial in Reunion island. Diabetes \& Metabolism ; 2012 ; 38 : 46-53.

16. BALCOU-DEBUSSCHE M \& DEBUSSCHE X. Hospitalisation for type 2 diabetes : the effects of the suspension of reality on patients' subsequent management of their condition. Qualitative Health Research ; 2009 ; 19(7) : 1100-1115.

17. DURKHEIM E. Education et sociologie. Paris Alcan ; 1922.

18. HOUSSAYE J. Théorie et pratiques de l'éducation scolaire : le triangle pédagogique. Bern : Editions Peter Lang ; 2000. 


\section{L'éducation thérapeutique : entre savoirs complexes, formateurs, apprenants hétérogènes et contextes pluriels}

19. TUPIN F. Les pratiques enseignantes et leurs contextes : des curricula aux marges d'action. Approche sociologique, regards pluriels. Habilitation à Diriger des Recherches : Université de Nantes ; 2006.

20. LAHIRE B. Portraits sociologiques. Dispositions et variations individuelles. Paris Nathan ; 2002.

21. BRIL B. Apprentissage et contexte. Intellectica ; 2002 ; 35 : 251-268.

22. GIBSON JJ. The Theory of Affordances. In : Perceiving, Acting, and Knowing, SHAW RE \& BRANSFORD J. (sous la direction de). Lawrence Erlbaum Associates, Hillsdale, NJ, 1977.

23. BALCOU-DEBUSSCHE M. Inégalités d'accès à une réflexion sur l'action par les pratiques scripturales: l'exemple de 4 formations professionnelles dans le domaine de la santé. Recherche et Formation ; 2003 ; 44 : 149-166.

24. BALCOU-DEBUSSCHE M. Écriture et formation professionnelle. L'exemple des professions de la santé. Lille Presses Universitaires du Septentrion ; 2004.

25. GIORDAN A. Apprendre ! Paris : Belin ; 1998.

26. MEIRIEU P. Le maître : de la transmission à la médiation. In : L'école et ses maîtres, Actes VII des entretiens Nathan. Paris Nathan ; 1997 : 79-97.

27. BAUTIER É. Pratiques langagières, pratiques sociales. De la sociolinguistique à la sociologie du langage. Paris L'Harmattan ; 1995.

28. GRAVOIS LEE R, GARVIN T. Moving from information transfer to information exchange in health and health care. Social Science and Medicine ; 2003 ; $56: 449-464$.

29. ROTHIER-BAUTZER E. Pratiques soignantes en mutation : de la lutte contre la maladie à la collaboration avec le patient. Revue Française de Pédagogie ; 2002 ; 138 : 39-50.

30. BALCOU-DEBUSSCHE M \& DEBUSSCHE X. Type 2 diabetes patient education in Reunion Island : Perceptions and needs of professionals in advance of the initiation of a primary care management network. Diabetes \& Metabolism ; 2008 ; 34(4) : 375-381.

31. HUGHES C. La fabrication d'un médecin. Les Sciences de l'éducation pour l'Ėre nouvelle ; 2003 ; 36(2) : 57-70.

32. LAHIRE B. L'homme pluriel. Les ressorts de l'action. Paris Nathan ; 1998.

33. BERGER D. La formation des acteurs de l'éducation à la santé : vers des praticiens réflexifs. In : La Formation des acteurs de l'éducation à la santé en milieu scolaire, JOURDAN D. (sous la direction de). Toulouse Éditions universitaires du Sud ; 2004.

34. FOUCAUD J \& BALCOU-DEBUSSCHE M. Former à l'éducation du patient : quelles compétences ? Paris : Éditions INPES ; 2008.

35. BURY M. Chronic illness as biographical disruption. Sociology of Health and IIIness ; 1982 ; 4 : 167-82.

36. FALKUM E \& FORDE R. Paternalism, patient autonomy, and moral deliberation in the physician-patient relationship attitudes with Norwegian physicians. Social Science and Medicine ; 2001 ; 52 : 239-248.
37. LAHIRE B. La construction de "l'autonomie " à l'école primaire : entre savoirs et pouvoirs. Revue française de Pédagogie 2001 ; 134 : 151-161.

38. LAUTIER N. Psychosociologie de l'éducation. Regard sur les situations d'enseignement. Paris Armand Colin ; 2001.

39. FESTINGER L. A theory of cognitive dissonance. Stanford University Press ; 1957.

40. TAJFEL H. La catégorisation sociale. In : Introduction à la psychologie sociale, MOSCOVICI S. (sous la direction de). Paris : Larousse ; 1972 ; 1.

41. DEBUSSCHE X \& BALCOU-DEBUSSCHE $M$. Combiner les approches biomédicale et psychosociale pour concevoir des situations d'apprentissage en éducation thérapeutique. Médecine des maladies Métaboliques ; 2011 ; 5 (2) : 180185.

42. BALCOU-DEBUSSCHE M. L'apport des sciences de l'éducation. Un exemple de situations d'apprentissage pour les personnes à risque cardiovasculaire. Santé Décision Management $2011 ; 13: 9-24$

43. DEBUSSCHE $X$, BALCOU-DEBUSSCHE M, BARANDERAKA NA, NDAYIRORERE S, LA HAUSSE DE LALOUVIERE $V$ \& NITUNGA $N$. Réseau d'éducation en santé communautaire pour la prévention des maladies cardiovasculaires et du diabète au Burundi : mise en place et premiers résultats. Global Health Promotion ; $2010 ; 17(68)$ : 67-75.

44. LORIG KR, SOBEL DS, LAURENT D, HOBBS M. Effect of a self-management program on patients with chronic disease. Effective Clinical Practice ; 2001 ; 4 : 256-262.

45. PEYROT M \& RUBIN R. Behavioral and psychosocial interventions in diabetes : a conceptual review. Diabetes Care ; 2007 ; 30(10) : 2433-2440

46. PEYROT M, RUBIN RR, FUNNELL MM, SIMINERIO LM. Access to diabetes self-management education : results of national surveys of patients, educators, and physicians. The Diabetes Educator ; 2009 ; 35(2) : 246-263.

47. HAWTHORNE K, ROBLES Y, CANNINGS JOHN R, EDWARDS AG. Culturally appropriate health education for type 2 diabetes mellitus in ethnic minority groups. Cochrane Database of Systematic Reviews ; 2008 ; Issue 3, CD006424.

48. BALCOU-DEBUSSCHE M. La littératie en santé : parler, comprendre, décider. In : La dimension langagière dans l'apprentissage et la formation, AURIAC-SLUSARCZYK E. (sous la direction de). Clermont-Ferrand, Presses Universitaires de I'Université Blaise Pascal ; 2012 ; 40-52.

49. DEBUSSCHE X, COLLIN F, FIANU A, BALCOU-DEBUSSCHE $M$, FOUET-ROSIERS I KOLECK M, FAVIER F. Structured self-management education maintained over two years in insufficiently controlled type 2 diabetes patients: the ERMIES randomised trial in Reunion Island. Cardiovascular Diabetology ; 2012 ; 11.

50. BILLON-DESCARPENTRIES J. Les concepts de perception et de représentations de la santé : intérêts et limites dans le domaine de l'éducation pour la santé. Recherche en soins nfirmiers ; $2003 ; 74: 31-37$. 